\title{
Promotion of Cervical Screening among Long-term Non- attendees by Human Papillomavirus Self-sampling
}

\author{
Avalon Ernstson', Ola Forslund ${ }^{2}$, Christer Borgfeldt ${ }^{1}$ \\ 1Department of Obstetrics and Gynecology, Skåne University Hospital, Lund University, ${ }^{2}$ Department of Medical Microbiology, \\ Laboratory Medicine Region Skåne, Lund University, Lund, Sweden
}

\begin{abstract}
Cervical cancer is preventable through gynecological screening. To promote participation among non-attending women, self-collected vaginal samples for detection of high-risk human papillomavirus (hr-HPV) is an option. The aims of this study were to investigate the response of self-collected vaginal samples for hr-HPV testing among long-term non-attendees, to explore the attendance at follow-up among HPV-positive women, and to analyze the prevalence of hr-HPV and severe cervical dysplasia or cancer among the responders. A vaginal self-sampling kit was sent to 19,766 women aged 30-70 years who had not provided a cervical screening sample for $\geq 7$ years in Skåne, Sweden. The self-sample was analyzed by the Aptima HPV mRNA assay (Hologic). Women testing positive for HPV were invited for follow-up. The response was 18.5\% (3,646/19,757). The prevalence of HPV mRNA was $11.3 \%$ (412/3,636). Among HPV-positive women, 85.7\% (353/412) attended follow-up, and of these, 44.8\% (158/353) had HPV in the cervical sample. The HPV mRNA test of self-samples showed a positive predictive value of $9.3 \%$ ([33/353], 95\% Cl = 6.5-12.9) for detection of cytologically severe dysplasia. Histologically severe dysplasia or cancer was detected in $0.88 \%$ ([32/3,636], 95\% $\mathrm{Cl}=$ 0.6-1.2) among responders, including two cervical- and one vaginal cancer. In conclusion, almost one fifth of the long-term non-attendees participated in self-collected vaginal hr-HPV sampling. The prevalence of histologically confirmed high grade squamous intraepithelial lesion or cervical cancer was not increased significantly compared to regularly screened women in Sweden. The relatively high HPV prevalence among the self-samples indicates the importance of diagnostic follow-up with cervical HPV testing and reflex-cytology of HPV-positive cases.
\end{abstract}

Key Words Uterine cervical neoplasms, Screening, Human papillomavirus, Self-sampling, Long-term non-attendees

\section{INTRODUCTION}

Sweden has a long history of prevention of cervical cancer since an organized cervical screening program was implemented in 1966 [1]. Because of this, the cervical cancer incidence decreased from 15.1 cases per 100,000 individuals in 1970 to 8.1 cases per 100,000 individuals in 2018 [2]. However, for a screening program to maintain effective, high coverage is essential and women that do not take part in cervical screening are at greater risk of cervical cancer [3]. It is estimated that almost two thirds of cervical cancer cases in Sweden occur in women who have not participated in the screening program at all or have not participated regularly [4]. With the introduction of testing for high-risk human papillomavirus (hr-HPV) in cervical screening, a vaginal human papillomavirus (HPV) sample collected by the woman herself has become a possibility. In Skåne, Sweden, where this study was coducted, the Aptima hr-HPV mRNA test is used on cervical- and self-samples. The Aptima mRNA analysis is based on the detection of the expressed oncogenes E6 and E7 and manifests increased specificity compared to HPV-DNA tests [5]. In a recent meta-analysis, vaginal self-collected samples analyzed by the Aptima hr-HPV assay demonstrated lower sensitivity compared to cervical samples [6]. Although, we have previously found a sensitivity for vaginal self-sampling similar to that of routine cytology [7] and there are studies that demonstrated substantial agreement between vaginal self-collected samples and cervical samples analyzed by the Aptima mRNA assay $[8,9]$. Self-samples can overcome most of the barriers to cervical screening that non-attendees experienced, such as feeling uncomfortable with a gynecological examination, lack of time, and experience of unfriendly health

Received November 21, 2020, Revised February 10, 2021, Accepted February 15, 2021

Correspondence to Avalon Ernstson, E-mail: avalon.ernstson@med.lu.se, https://orcid.org/0000-0001-9559-3379

Check for updates 
workers [10]. Furthermore, offering self-samples to screening non-attendees is in accordance with the national guidelines of Sweden [1], although only seven of 21 regions had implemented these guidelines in September, 2020 [11]. In addition, self-sampling is generally accepted among women [12,13]. Previously we have observed responses to self-sampling among screening non-attendees of between $13 \%$ and $43 \%$, the latter among older women (69 to 70 years) [14,15]. Other studies have shown a response rate among women with no cervical sample for 5 to 9 years, aged 30 to 65 years old, of $15 \%$ to $58 \%$ [10,16-19].

We attempted to further evaluate the effects of offering HPV self-samples to long-term non-attendees in a current screening program as a potential method to promote participation in cervical screening. The aims of this study were: to investigate the response rate of a free of charge self-collected vaginal hr-HPV sample sent to women who had not provided a cervical smear for $\geq 7$ years; to explore the attendance rate at follow-up among women positive for HPV in the self-collected vaginal sample; and to analyze the prevalence of hr-HPV and severe cervical dysplasia or cancer among the responders. This study was also aimed to investigate the distribution of responses and HPV positivity among different age categories.

\section{MATERIALS AND METHODS}

During spring 2018, all women with registered home address in the county of Skåne, Sweden, aged 30 to 70 years, who had not provided a cervical smear for $\geq 7$ years, were identified through the southern regional cervical screening registry. The registry contains information on all smears taken in the region, whether organized or spontaneously taken. On May 22 and 28, 2018, 19,766 women were offered a free of charge vaginal HPV self-sampling kit as previously described [14]. All vaginal self-samples returned no later than May 31, 2019 were included in the study. No reminder was communicated if the kit was not returned. Follow-up of the results was registered until October 2, 2019 with a follow-up time of between 4 to 17 months. Analysis of the self-samples was carried out using the Aptima HPV mRNA assay (Hologic, San Diego, CA, USA) on a Panther instrument, according to the manufacturer's instructions. The Aptima HPV assay detects HPV mRNA from $14 \mathrm{hr}-\mathrm{HPV}$ types (16, 18, 31, 33, 35, 39, 45, $51,52,56,58,59,66$, and 68).

In the case of a negative HPV test result, the women were returned to the organized screening program and informed by means of an automatically generated letter from the Department of Laboratory Medicine. If the test result was invalid, the women were informed by a letter and asked to contact a midwife health station to arrange for a cervical HPV sample to be taken. Women with a positive HPV test result received a letter from the nearest midwife health station with information about the presence of hr-HPV and an invitation to have a clinical follow-up examination by the midwife. The follow-up examination consisted of a cervical sample for cytological analysis as well as Aptima HPV mRNA testing. A reminder letter was sent if the woman did not attend her follow-up. If the woman had still not attended her follow-up examination in May to June 2019, a second reminder letter was sent. In case of abnormal cytological results in the follow-up examination, the women were referred for colposcopy according to regional guidelines [1]. In the case of several diagnoses at the follow-up, the worst cytology/histology diagnosis was used in this study. In Sweden, the Bethesda terminology is used for classification of cytological and histological findings [1].

\section{Statistical analyses}

Statistical comparisons were based on the binomial distribution and $95 \% \mathrm{Cl}$ were given. Microsoft ${ }^{\circledR}$ Excel, Version 15.30 (Microsoft, Redmond, WA, USA) was used on a Mac computer. Comparisons were made using a Pearson chi-square test. The comparison was two-sided and $P$-values less than 0.05 were considered statistically significant. IBM SPSS statistics version 26 (IBM Corp., Armonk, NY, USA) was used for calculations.

\section{Ethical approval}

This study was approved by the Regional Ethical Review Board, Lund (DNR 2013-390 [2013-06-19] with amendment 2018-466 [2018-05-18]). Returning the self-sample was de-

19,766 self-collected vaginal hr-HPV kits were sent to women with no cervical smear for 7 years or more

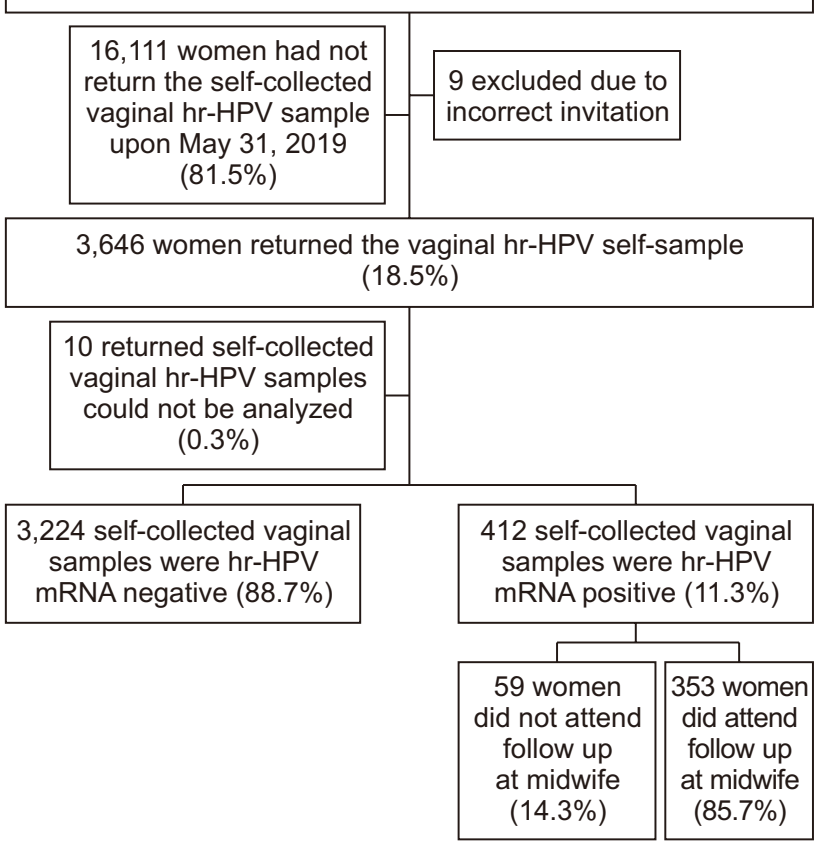

Figure 1. Flow-chart showing study population for invitation to self-collected vaginal hr-HPV sampling. Hr-HPV, high-risk human papillomavirus. 
fined as the woman's consent to participate.

\section{RESULTS}

Out of the 19,766 self-sampling kits sent to long-term non-attendees, nine were excluded due to an incorrect invitation since these women had taken a cervical smear in the last two months before the self-sampling offer (Fig. 1). The remaining 19,757 women were correctly offered a self-sampling kit. Out of these, the response rate was $18.5 \%([3,646 / 19,757], 95 \%$ $\mathrm{Cl}=17.9$ to 19.0 ) (Fig. 1). The mean age of the responding women was 52.6 years (SD 9.8, median 54.0, range 30 to 71 [women of age 71 years turned 71 during the follow-up time]). The age group 30 to 39 years had a higher response rate of $21.9 \%$, while the response rate for women aged $>40$ years was $18.1 \%(P<0.001)$ (Table 1$)$. Of the responding women, the majority $(63.5 \%)$ returned their self-sample within one month after the offer. After six months, $95.5 \%$ of the responding women had returned their self-sample (Fig. 2). Out of the returned self-samples, $0.3 \%([10 / 3,646], 95 \% \mathrm{Cl}=0.1$ to 0.5$)$ could not be analyzed (Fig. 1).

The HPV prevalence was $11.3 \%([412 / 3,636], 95 \% \mathrm{Cl}=$ 10.3 to 12.4) (Fig. 1). The mean age of the HPV-positive women was 51.2 years (SD 10.2, median 53.0, range 30 to 71). A higher HPV prevalence could be observed among women 30 to 49 years old in comparison with those aged 50 to 71 years $(P=0.002)$. The lowest HPV prevalence was in the age group 50 to 59 years $(9.8 \%)$ and the highest in the age group 30 to 39 years (14.5\%) (Table 1). Of the women positive for HPV in the self-sample, $85.7 \%$ ([353/412], 95\% Cl = 81.9 to 88.9) attended follow-up (Fig. 1). The median number of days between the laboratory receiving the returned self-sample and the woman participating in the follow-up was 38 days (range 12 to 418 , interquartile range 32 ). At the follow-up, HPV was detected in $44.8 \%$ ([158/353], 95\% Cl $=39.5$ to 50.1 ) of the cervical samples (Table 2). There was no statistical difference in cervical HPV positivity rate correlated to follow-up time after positive HPV self-sample (Table 3).

\section{Cervical dysplasia and cervical cancer}

The presence of severe cervical dysplasia (high grade squa- mous intraepithelial lesion [HSIL] or atypical squamous cells that cannot exclude HSIL [ASC-H]) or cancer detected by cytology was $9.3 \%$ ([33/353], $95 \% \mathrm{Cl}=6.5$ to 12.9$)$, representing $0.91 \%([33 / 3,636], 95 \% \mathrm{Cl}=0.6$ to 1.3$)$ in the whole group of responders. In accordance, the HPV mRNA test of self-samples showed a positive predictive value (PPV) of $9.3 \%$ ([33/353], $95 \% \mathrm{Cl}=6.5$ to 12.9 ) for detection of cytological severe dysplasia (Table 2). At follow-up, the HPV mRNA test performed on cervical samples showed a PPV of $20.9 \%$ ([33/158], $95 \% \mathrm{Cl}=14.8$ to 28.1 ) for detection of cytological severe dysplasia (Table 2). The histologically confirmed prevalence of HSIL or cancer was $9.1 \%$ ([32/353], 95\% Cl $=6.3$ to 12.6) among women attending the follow-up and in the whole group of responders it was $0.88 \%([32 / 3,636], 95 \% \mathrm{Cl}=0.6$ to 1.2), including two cervical- and one vaginal cancer (Table 2). Among the 32 women with histologically confirmed HSIL or cancer, there was an average of 14.5 years (SD 6.0, median 12.5 , range 7 to 27 ) since the last registered cervical smear in our registers, and $84.4 \%$ had benign cytology at their last cervical smear. The cervical cancer cases consisted of one adenosquamous carcinoma FIGO (The International Federation of Gynecology and Obstetrics) stage IA1 and one squamous

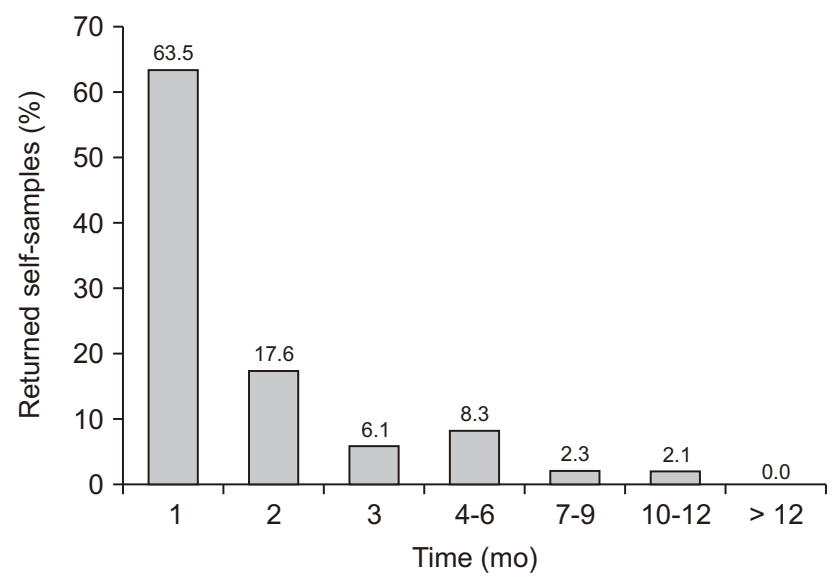

Figure 2. Time in months between offered and returned vaginal hr-HPV mRNA self-collected sample. Hr-HPV, high-risk human papillomavirus.

Table 1. Response rate and HPV positivity in vaginal self-collected HPV mRNA analyzes stratified by age groups

\begin{tabular}{|c|c|c|c|c|c|c|}
\hline \multirow{2}{*}{$\begin{array}{l}\text { Age group } \\
\text { (yr) }\end{array}$} & \multirow{2}{*}{$\begin{array}{l}\text { Number invited } \\
\text { to self-sampling }\end{array}$} & \multicolumn{2}{|c|}{ Response rate } & \multirow{2}{*}{$\begin{array}{c}\text { HPV } \\
\text { positive (n) }\end{array}$} & \multirow{2}{*}{$\begin{array}{c}\text { HPV } \\
\text { negative (n) }\end{array}$} & \multirow{2}{*}{$\begin{array}{c}\text { HPV prevalence, } \\
\%(95 \% \mathrm{Cl})\end{array}$} \\
\hline & & Number & $\%(95 \% \mathrm{Cl})$ & & & \\
\hline $30-39$ & 2,079 & 455 & $21.9^{a}(20.1-23.7)$ & 66 & 388 & $14.5(11.4-18.1)$ \\
\hline $40-49$ & 4,592 & 814 & $17.7(16.6-18.9)$ & 106 & 706 & $13.1(10.8-15.6)$ \\
\hline $50-59$ & 7,937 & 1,397 & $17.6(16.8-18.5)$ & 136 & 1,257 & $9.8(8.3-11.4)$ \\
\hline $60-71$ & 5,149 & 980 & $19.0(18.0-20.1)$ & 104 & 873 & $10.6(8.8-12.8)$ \\
\hline Total & 19,757 & 3,646 & $18.5(17.9-19.0)$ & $412^{b}$ & $3,224^{b}$ & $11.3(10.3-12.4)$ \\
\hline
\end{tabular}

HPV, human papillomavirus. ${ }^{\mathrm{a}} \mathrm{P}$-value $30-39$ years vs. others $<0.001 .{ }^{\mathrm{b}}$ After exclusion of 10 returned self-collected samples that could not be analyzed. 
Table 2. Results of the 353 women with hr-HPV mRNA positive self-samples attending the follow-up examination including status of hr-HPV at cervix, cytology- and histological assessments

\begin{tabular}{|c|c|c|}
\hline Cytology & Hr-HPV status cervix & Histology \\
\hline \multirow[t]{2}{*}{ No cytologi ${ }^{a}(n=10)$} & Positive: 0 (0) & - \\
\hline & Negative: 10 (100) & $\begin{array}{l}\text { No histology: } 9 \\
\text { Benign: } 1\end{array}$ \\
\hline \multirow[t]{2}{*}{ Benign $(n=225)$} & Positive: 52 (23.1) & $\begin{array}{l}\text { No histology: } 51 \\
\text { Benign: } 1\end{array}$ \\
\hline & Negative: 173 (76.9) & No histology: 173 \\
\hline \multirow[t]{2}{*}{ ASCUS $(n=46)$} & Positive: 35 (76.1) & $\begin{array}{l}\text { No histology: } 15 \\
\text { Benign: } 14 \\
\text { LSIL: } 5 \\
\text { HSIL: } 1\end{array}$ \\
\hline & Negative: 11 (23.9) & $\begin{array}{l}\text { No histology: } 7 \\
\text { Benign: } 4\end{array}$ \\
\hline \multirow[t]{2}{*}{ LSIL ( $n=39)$} & Positive: 38 (97.4) & $\begin{array}{l}\text { No histology: } 17 \\
\text { Benign: } 12 \\
\text { LSIL: } 7 \\
\text { HSIL: } 2\end{array}$ \\
\hline & Negative: 1 (2.6) & No histology: 1 \\
\hline \multirow[t]{2}{*}{ ASC-H $(n=7)$} & Positive: $7^{c}(100)$ & $\begin{array}{l}\text { Benign: } 1 \\
\text { LSIL: } 1 \\
\text { HSIL: } 5\end{array}$ \\
\hline & Negative: $0(0)$ & - \\
\hline \multirow[t]{2}{*}{ HSIL $(n=26)$} & Positive: $26^{c}(100)$ & $\begin{array}{l}\text { No histology: } 1 \\
\text { Benign: } 1 \\
\text { HSIL: } 21 \\
\text { Cancer: } 3^{\text {b }}\end{array}$ \\
\hline & Negative: $0(0)$ & - \\
\hline Total $(n=353)$ & $\begin{array}{l}\text { Total positive: } \\
158 \text { (44.8) } \\
\text { Total negative: } \\
195(55.2)\end{array}$ & $\begin{array}{l}\text { Total no histology: } \\
274 \\
\text { Total benign: } 34 \\
\text { Total LSIL: } 13 \\
\text { Total HSIL: } 29^{\mathrm{d}} \\
\text { Total cancer: } 3^{\mathrm{d}}\end{array}$ \\
\hline
\end{tabular}

Values are presented as number only or number (\%). Hr-HPV, high-risk human papillomavirus; HPV, human papillomavirus; ASCUS, atypical squamous cells of undetermined significance; LSIL, low grade squamous intraepithelial lesion; ASC-H, atypical squamous cells cannot exclude HSIL; HSIL, high grade squamous intraepithelial lesion. ${ }^{\text {aT }}$ The reason for no cytological diagnosis was due to insufficient material in six of the test samples and no cytology taken for four samples. ${ }^{\mathrm{b}} \mathrm{Tw}$ cases of cervical cancer, one case of vaginal cancer. ${ }^{\circ}$ For calculation of positive predictive value (PPV) for self-samples the frequency of ASC-H and HSIL $(n=33)$ was used as the numerator and the sum of HPV-positive self-samples $(n=353)$ was denominator: $9.3 \%$ ([33/353], $95 \% \mathrm{Cl}=$ 6.5-12.9) for detection of cytological severe dysplasia. The HPV mRNA test performed on cervical samples at follow-up showed a PPV of $20.9 \%([33 / 158], 95 \% \mathrm{Cl}=14.8-28.1)$ for detection of cytological severe dysplasia. ${ }^{\mathrm{d}}$ The histologically confirmed prevalence of $\mathrm{HSIL}$ or cancer was $9.1 \%$ ([32/353], 95\% Cl $=6.3-$ 12.6) among women attending the follow-up.

cell carcinoma FIGO stage IIB, and their last cervical smears were registered $\geq 11$ years ago with benign cytology.

\section{DISCUSSION}

Among long-term non-attendees of cervical screening in the county of Skåne, southern Sweden, we observed a response rate of $18.5 \%$ by opt-out self-sampling devices. The response rate was highest among the youngest participants aged 30 to 39 years. Among the responders, $11.3 \%$ were HPV mRNA positive. The HPV mRNA test of self-samples showed a PPV of $9.3 \%$ for detection of cytological severe dysplasia. The compliance to follow-up was $85.7 \%$ of vaginally HPV-positive women and $44.8 \%$ had detectable HPV mRNA in the cervical sample. Histologically confirmed severe dysplasia or cancer was detected among $0.88 \%$ of the responders, including two cases of cervical cancer and one of vaginal cancer.

We found a response rate of $18.5 \%$ which was higher than the $13.2 \%(P<0.001)$ observed in our previous study with the same inclusion criteria but a smaller study population of 6,023 women [14]. The difference in responses between the two studies cannot be explained by a shorter inclusion-time of six months in the previous study since the corresponding response before six months in the present study was $17.7 \%$ $(3,498 / 19,757)(P<0.001)$. The increased response rate might have been due to a rise of acceptance of self-sampling in the society. The response rate of $18.5 \%$ in the present study is in agreement with $19.2 \%$, reported in a recent meta-analysis investigating strategies to reach underscreened or not screened women by offering self-samples [6]. We found that the youngest age group, 30 to 39 years, had a significantly higher response rate of $21.9 \%$ compared to the other age groups. Other studies have found no age difference regarding participation rate [16] or a slightly higher participation rate among the oldest age groups [20,21].

The HPV mRNA prevalence in this study of vaginal self-samples was $11.3 \%$, which is significantly higher compared to the HPV mRNA prevalence of $7 \%$ among cervical HPV testing in women $\geq 30$ years in the county of Skåne who had undergone regular cervical screening $(P<0.001)$ [22]. Previous studies from Sweden have found that HPV prevalence among self-samples varies between $6.7 \%$ and $26 \%$ among women who had not provided a cervical smear for $>6$ years $[10,14,16,17,19,20]$. In the meta-analysis by Arbyn et al. [6] the HPV prevalence in self-samples among underscreened or not screened women was found to be between $6.0 \%$ and $29.4 \%$, where the pooled data from 22 trials showed a prevalence of $11.1 \%$, which is similar to our results. A decreasing HPV prevalence with increasing age could be seen in our study, which is in concordance with findings from several other self-sampling studies $[16,20,21,23]$ and with the organized cervical screening in the county of Skåne [22].

The cytologically confirmed prevalence of severe dysplasia or cancer in the whole group of responders in our study was similar to the organized screening in the county of Skåne in the year $2016(0.91 \%$ in our study vs. $0.71 \%$ in Skåne 2016, $P=0.171$ ) [22]. In comparisons with the entire organized screening program in Sweden 2017, those with severe dysplasia in cytology had a similar prevalence (cytology: $0.91 \%$ in our study vs. $1.2 \%$ in Sweden, $P=0.099$ ) but the 
Table 3. Positivity rate of cervical HPV at the follow-up stratified for days between when the laboratory received the returned self-sample and when the woman participated in the follow-up

\begin{tabular}{|c|c|c|c|c|c|c|c|}
\hline \multirow{2}{*}{ Days to follow-up } & \multicolumn{3}{|c|}{ Cervical HPV positive } & \multicolumn{3}{|c|}{ Cervical HPV negative } & \multirow{2}{*}{ Total $(n)$} \\
\hline & Number & $\%$ & $95 \% \mathrm{Cl}$ & Number & $\%$ & $95 \% \mathrm{Cl}$ & \\
\hline $12-30$ & 45 & 37.8 & $29.1-47.2$ & 74 & 62.2 & $52.8-70.9$ & 119 \\
\hline $31-60$ & 73 & 49.0 & $40.7-57.3$ & 76 & 51.0 & $42.7-59.3$ & 149 \\
\hline $61-90$ & 26 & 50.0 & $35.8-64.2$ & 26 & 50.0 & $35.8-64.2$ & 52 \\
\hline $91-180$ & 7 & 46.7 & 21.3-73.4 & 8 & 53.3 & $26.6-78.7$ & 15 \\
\hline $181-365$ & 4 & 33.3 & $9.9-65.1$ & 8 & 66.7 & $34.9-90.1$ & 12 \\
\hline$>365$ & 3 & 50.0 & $11.8-88.2$ & 3 & 50.0 & $11.8-88.2$ & 6 \\
\hline
\end{tabular}

HPV, human papillomavirus.

histological prevalence of severe dysplasia or cancer in the entire group of responders was lower in our study (histology: $0.88 \%$ in our study vs. $1.6 \%$ in Sweden, $P<0.001$ ) [24]. The reason for the lower histological prevalence of severe dysplasia cannot be explained by a low proportion of histological specimens since $97.0 \%$ of women with cytological severe dysplasia had a histological specimen taken in our study. Interestingly, among the severe dysplasia cases in our study, we noted that the mean time since the last cervical smear was around three to five times longer than the recommended screening interval, and that the majority (84\%) of the women had benign cytology at their last previous cervical smear. Since we do not know when the HPV-positive women acquired their infection, it is possible that a proportion of the infections were relatively recently acquired and therefore no severe dysplasia or cancer had yet been developed. In support of such a speculation, we observed that $23.1 \%(52 / 225)$ of the normal cytology samples were HPV-positive at the follow-up examination, which is a considerably higher HPV prevalence compared with the organized cervical screening program in our county where only $3.9 \%$ of the normal cytology samples were found HPV-positive [22]. If focusing solely on the prevalence of cervical cancer in our study, it was similar to the prevalence found in the organized cervical screening program in entire Sweden in $2017(0.055 \%$ in our study vs. $0.061 \%$ in Sweden, $P=0.880$ ) [24]. Other studies have found that the cervical cancer prevalence varies between $0 \%$ and $1.0 \%$ among non-attendees who had not provided a cervical smear for 5 to 9 years [10,14,18,19,25], indicating a large variation in cervical cancer prevalence among screening non-attendees.

Concerning the implementation of self-sampling as a method to reach screening non-attendees, it is crucial that the women are well informed about the necessity of a follow-up examination in the case of HPV positivity. Non-attendees have reported a reluctance to have a gynecological examination [10], which might become an obstacle when it comes to participation at the follow-up. In this study, $85.7 \%$ of the HPV mRNA-positive women attended the follow-up examination, which is similar to the results from our previous study with a participation rate of $83.5 \%$ [14]. The meta-analysis by Arbyn et al. [6] found an average participation rate of $80.6 \%$ for follow-up. Women that did not attend the follow-up in our study were reminded to do so by letter. However, in our previous study, we tried to reach follow-up non-attendees through a telephone reminder, although only one out of 13 women was reached [14]. An alternative strategy to reach self-sampled HPV-positive women is through sending repeated HPV self-sampling kits as described in a study by Gustavsson et al. [26]. They found that $29 \%$ of the initially HPV-positive women had cleared their HPV infection after an average of 4.4 months. A second self-sample sent to HPV-positive women would lower the burden on the health-care system. In the study by Gustavsson et al. [26], 97\% of the initially HPV-positive women had a second test ( $90 \%$ through the second HPV self-sample and $7 \%$ through clinician follow-up).

Notably, among the self-sample HPV-positive women only $44.8 \%$ had detectable HPV mRNA in the cervical sample at follow-up. However, all women with histologically low grade squamous intraepithelial lesion (LSIL), HSIL or cancer were HPV mRNA-positive in the cervical sample at follow-up, indicating that reflex-cytology evaluation should be limited to only HPV-positive women at follow-up. We observed that the HPV mRNA test of self-samples showed a PPV of $9.3 \%$ for detection of cytological severe dysplasia. This is in agreement with our organized primary HPV screening program for the year 2017, where the corresponding PPV of cervical samples was 9.7\% (379/3897) [22]. However, in the present study HPV mRNA testing at follow-up of cervical samples demonstrated an increased PPV of $20.9 \%$ for cytological severe dysplasia. Thus, in our county, within a selected group of long-term non-attendees with HPV mRNA in self-samples about a fifth of those with HPV mRNA in cervical samples at follow-up demonstrated severe cytological dysplasia. Although that only $44.8 \%$ had detectable HPV in the cervix sample at follow-up, this is similar to an HPV positivity of $47 \%$ observed among non-attendees at follow-up in our previous study [14]. One reason for the relatively low HPV prevalence at follow-up was that benign cytology was predominant $(65.6 \%, 225 / 343)$, where most of the samples had no detectable HPV $(76.9 \%$, 173/225). It is possible that clearance of HPV could play a role for the modest HPV detection rate at follow-up since 
studies have found HPV-positivity of $59 \%$ to $73 \%$ at renewed vaginal HPV testing of self-sampled HPV positive women $[16,26,27]$. Furthermore, Bulkmans et al. [28] found a clearance rate of hr-HPV at six and 18 months of $43 \%$ and $65 \%$ respectively among women with normal cytology at baseline. However in our study, we did not observe an increased HPV clearance rate with increased time to follow-up. Another aspect of HPV-positive self-sampled women, who are HPV negative in the cervical sample, is the possibility for a different flora of HPV in the vagina compared to that of the cervix. Coorevits et al. [29] found that hr-HPV was detected more frequently in vaginal samples compared with cervical samples; however, when including the probable hr-HPV types $(26,53$, and 66$)$, there was no difference between vaginal and cervical samples. Castle et al. [30] found no difference in prevalence regarding carcinogenic HPV in vaginal and cervical specimens in a study of 332 women. In the meta-analysis, Arbyn et al. [6] concluded that there was lower sensitivity for hr-HPV mRNA assay using vaginal self-samples compared to that of cervical clinician-taken samples, but other studies have found a substantial agreement between vaginal self-samples and clinician-taken cervical samples analyzed for hr-HPV mRNA $[8,9]$. Overall, more research is needed to clarify differences in HPV mRNA positivity between vaginal self-samples and cervical samples.

The main strengths of this study were the large study population and that the study was performed as part of the current population-based cervical screening program which enhance the external validity when it comes to the aim of investigating the response rate of a self-sample among screening long-term non-attendees. But, since 3,646 responders was not representative of 19,757 non-attendees the external validity could not be guaranteed and the response rate to free of charge vaginal self-samples for HPV analyses may differ in other populations in Sweden and other high-income countries. In Sweden, $5 \%$ to $6 \%$ of women aged 40 to 60 years old have had a total hysterectomy [31], which is a criterion for exclusion from cervical screening invitations [1]. In this study, we used the same registers as the organized screening program; therefore, previous total hysterectomy was not a specific exclusion criterion in the study. However, during a random control, we found that several included women had a previous total hysterectomy, which may have affected the participation rate towards a lower response rate and shows that women with no cervix are incorrectly invited to cervical screening. One inclusion criterion for the study was that the women should not have provided a cervical sample for $\geq$ 7 years. However, we only had information about previous cervical samples if collected in the Skåne region, which is a limitation. Another limitation was that the invitation and instructions for the self-sampling procedure were only provided in Swedish, and no reminder was sent. In the future information in other languages should be provided and a reminder to non-responders should be sent to increase participation.
In conclusion, almost one fifth of the long-term non-attendees participated in self-collected vaginal hr-HPV sampling. The prevalence of histological HSIL or cervical cancer was not increased compared to regularly screened women. However, the relatively high HPV prevalence indicates the importance of diagnostic follow-up with repeated cervical HPV testing and reflex-cytology of HPV positive cases.

\section{ACKNOWLEDGMENTS}

The Authors would like to thank the personnel at the Department of Medical Microbiology and Clinical Pathology, Laboratory Medicine, Region Skåne, Lund, Sweden.

This study was supported by Region Skåne Research Funds.

\section{CONFLICTS OF INTEREST}

Ola Forslund has received a speech honorarium from Hologic and his laboratory department (Laboratory Medicine, Region Skåne, Lund) has ongoing contracts with Hologic. Christer Borgfeldt has received a grant from Hologic Inc 2018 to perform HPV studies. None of the other authors have any conflicts of interest to declare. Hologic Inc. did not have any influence on the study design, statistical analyses, or manuscript writing.

\section{ORCID}

Avalon Ernstson, https://orcid.org/0000-0001-9559-3379

Ola Forslund, https://orcid.org/0000-0001-6293-7286

Christer Borgfeldt, https://orcid.org/0000-0003-2267-8325

\section{REFERENCES}

1. Regional Cancer Centers (RCC). [Cervical cancer prevention- National care program]. https://www.cancercentrum. se/globalassets/vara-uppdrag/prevention-tidig-upptackt/ gynekologisk-cellprovskontroll/vardprogram/nationellt-vardprogramcervixcancerprevention.pdf. Accessed September 9, 2019. Swedish.

2. National Board of Health and Welfare. [Statistical database for cancer]. https://sdb.socialstyrelsen.se/if_can/val.aspx. Accessed September 9, 2019. Swedish.

3. Andrae B, Kemetli L, Sparén P, Silfverdal L, Strander B, Ryd W, et al. Screening-preventable cervical cancer risks: evidence from a nationwide audit in Sweden. J Natl Cancer Inst 2008;100:622-9.

4. Regional Cancer Centers (RCC). [Cervical cancer and vaginal cancer- National care program]. https://kunskapsbanken. cancercentrum.se/globalassets/cancerdiagnoser/gynekologi/ livmoderhalscancer/varprogram/nationellt-vardprogramlivmoderhalscancer-cervixcancer-vaginalcancer.pdf. Accessed September 12, 2019. Swedish.

5. Austin RM. Technology matters: the clinical utility of HPV mRNA testing compared to DNA testing. https://hologiced.com/wp- 
content/uploads/2019/01/Austin-supplement.pdf. Accessed September 25, 2019.

6. Arbyn M, Smith SB, Temin S, Sultana F, Castle P; Collaboration on Self-Sampling and HPV Testing. Detecting cervical precancer and reaching underscreened women by using HPV testing on self samples: updated meta-analyses. BMJ 2018;363:k4823.

7. Asciutto KC, Ernstson A, Forslund O, Borgfeldt C. Self-sampling with HPV mRNA analyses from vagina and urine compared with cervical samples. J Clin Virol 2018;101:69-73.

8. Des Marais AC, Zhao Y, Hobbs MM, Sivaraman V, Barclay L, Brewer NT, et al. Home self-collection by mail to test for human papillomavirus and sexually transmitted infections. Obstet Gynecol 2018;132:1412-20.

9. Chernesky M, Jang D, Gilchrist J, Elit L, Lytwyn A, Smieja M, et al. Evaluation of a new APTIMA specimen collection and transportation kit for high-risk human papillomavirus E6/E7 messenger RNA in cervical and vaginal samples. Sex Transm Dis 2014;41:365-8.

10. Darlin L, Borgfeldt C, Forslund O, Hénic E, Hortlund M, Dillner $\mathrm{J}$, et al. Comparison of use of vaginal HPV self-sampling and offering flexible appointments as strategies to reach long-term non-attending women in organized cervical screening. J Clin Virol 2013;58:155-60.

11. Regional Cancer Centers (RCC). [Status of the introduction of the cervical cancer prevention program]. https://www. cancercentrum.se/samverkan/vara-uppdrag/prevention-ochtidig-upptackt/gynekologisk-cellprovskontroll/vardprogram/statusfor-inforandet/. Accessed September 17, 2020. Swedish.

12. Virtanen $A$, Nieminen $P$, Niironen $M$, Luostarinen $T$, Anttila $A$. Self-sampling experiences among non-attendees to cervical screening. Gynecol Oncol 2014;135:487-94.

13. Nelson EJ, Maynard BR, Loux T, Fatla J, Gordon R, Arnold LD. The acceptability of self-sampled screening for HPV DNA: a systematic review and meta-analysis. Sex Transm Infect 2017;93:56-61.

14. Ernstson A, Urdell A, Forslund O, Borgfeldt C. Cervical cancer prevention among long-term screening non-attendees by vaginal self-collected samples for hr-HPV mRNA detection. Infect Agent Cancer 2020;15:10.

15. Ernstson A, Asciutto KC, Sturesson J, Norén J, Forslund O, Borgfeldt C. Detection of HPV mRNA in self-collected vaginal samples among women at 69-70 years of age. Anticancer Res 2019;39:381-6.

16. Sanner K, Wikström I, Strand A, Lindell M, Wilander E. Selfsampling of the vaginal fluid at home combined with high-risk HPV testing. Br J Cancer 2009;101:871-4.

17. Broberg G, Gyrd-Hansen D, Miao Jonasson J, Ryd ML, Holtenman M, Milsom I, et al. Increasing participation in cervical cancer screening: offering a HPV self-test to long-term non-attendees as part of RACOMIP, a Swedish randomized controlled trial. Int J Cancer 2014;134:2223-30.

18. Gök M, Heideman DA, van Kemenade FJ, Berkhof J, Rozendaal
L, Spruyt JW, et al. HPV testing on self collected cervicovaginal lavage specimens as screening method for women who do not attend cervical screening: cohort study. BMJ 2010;340:c1040.

19. Wikström I, Stenvall H, Wilander E. Attitudes to self-sampling of vaginal smear for human papilloma virus analysis among women not attending organized cytological screening. Acta Obstet Gynecol Scand 2007;86:720-5.

20. Stenvall H, Wikström I, Wilander E. High prevalence of oncogenic human papilloma virus in women not attending organized cytological screening. Acta Derm Venereol 2007;87:243-5.

21. Enerly E, Bonde J, Schee K, Pedersen H, Lönnberg S, Nygård $M$. Self-sampling for human papillomavirus testing among nonattenders increases attendance to the Norwegian Cervical Cancer Screening Programme. PLoS One 2016;11:e0151978.

22. Lindroth Y, Borgfeldt C, Thorn G, Bodelsson G, Forslund O. Population-based primary HPV mRNA cervical screening compared with cytology screening. Prev Med 2019;124:61-6.

23. Tranberg M, Bech BH, Blaakær J, Jensen JS, Svanholm $\mathrm{H}$, Andersen B. Preventing cervical cancer using HPV selfsampling: direct mailing of test-kits increases screening participation more than timely opt-in procedures- a randomized controlled trial. BMC Cancer 2018;18:273.

24. Swedish National Cervical Screening Registry. Ouality indicatorscrossreference cytology- histopathology organized screening. http://www.nkcx.se/Kv2_all_org.htm. Accessed October 4, 2019.

25. Szarewski A, Cadman L, Mesher D, Austin J, Ashdown-Barr L, Edwards R, et al. HPV self-sampling as an alternative strategy in non-attenders for cervical screening- a randomised controlled trial. Br J Cancer 2011;104:915-20.

26. Gustavsson I, Aarnio R, Berggrund M, Hedlund-Lindberg J, Strand AS, Sanner K, et al. Randomised study shows that repeated self-sampling and HPV test has more than two-fold higher detection rate of women with CIN2+ histology than Pap smear cytology. Br J Cancer 2018;118:896-904.

27. Gyllensten U, Sanner K, Gustavsson I, Lindell M, Wikström I, Wilander E. Short-time repeat high-risk HPV testing by self-sampling for screening of cervical cancer. $\mathrm{Br} \mathrm{J}$ Cancer 2011;105:694-7.

28. Bulkmans NW, Berkhof J, Bulk S, Bleeker MC, van Kemenade FJ, Rozendaal L, et al. High-risk HPV type-specific clearance rates in cervical screening. Br J Cancer 2007;96:1419-24.

29. Coorevits L, Traen A, Bingé L, Van Dorpe J, Praet M, Boelens $\mathrm{J}$, et al. Are vaginal swabs comparable to cervical smears for human papillomavirus DNA testing? J Gynecol Oncol 2018;29:e8.

30. Castle PE, Rodriguez AC, Porras C, Herrero R, Schiffman M, Gonzalez P, et al. A comparison of cervical and vaginal human papillomavirus. Sex Transm Dis 2007;34:849-55.

31. The Swedish National Quality Register of Gynecological Surgery (Gynop). http://www.gynop.se/for-kliniker/arsrapporter. Accessed March 18, 2020. 